\title{
Positive Association between GCKR rs780093 Polymorphism and Coronary Heart Disease in the Aged Han Chinese
}

\author{
Jiangfang Lian, ${ }^{1}$ Jian Guo, ${ }^{1,2}$ Zhikui Chen,, Qingjun Jiang, ${ }^{1}$ Huadan Ye, ${ }^{2}$ Xiaoyan Huang, \\ Xi Yang, ${ }^{1}$ Yanna Ba, ${ }^{1,2}$ Jianqing Zhou, ${ }^{1}$ and Shiwei Duan ${ }^{2}$ \\ ${ }^{1}$ Ningbo Medical Center, Lihuili Hospital, Ningbo University, Ningbo, Zhejiang 315041, China \\ ${ }^{2}$ Zhejiang Provincial Key Laboratory of Pathophysiology, Ningbo University, Ningbo, Zhejiang 315211, China
}

Correspondence should be addressed to Jianqing Zhou; ghw212@hotmail.com and Shiwei Duan; duanshiwei@nbu.edu.cn

Received 22 September 2013; Revised 31 October 2013; Accepted 31 October 2013

Academic Editor: Stamatios Theocharis

Copyright (C) 2013 Jiangfang Lian et al. This is an open access article distributed under the Creative Commons Attribution License, which permits unrestricted use, distribution, and reproduction in any medium, provided the original work is properly cited.

Objective. Previous studies have confirmed that GCKR rs780093 polymorphism is associated with triglyceride (TG), a known risk factor of coronary heart disease (CHD). The goal of our study is to explore the association of GCKR rs780093 polymorphism with CHD in Han Chinese population. Methods and Results. A total of $568 \mathrm{CHD}$ cases and 494 non-CHD controls were enrolled in the current case-control study. Genotyping was done using melting temperature shift (Tm-shift) approach. Our results also showed that GCKR rs780093 polymorphism was significantly associated with TG level $(P=0.0016)$. Although there was no significant association between cases and controls $(P>0.05)$, a breakdown analysis by age yielded a significant association of GCKR rs780093 polymorphism with CHD in individuals aged 65 and older (genotype: $\chi^{2}=6.86 ; \mathrm{df}=2 ; P=0.03$; allele: $\chi^{2}=4.11 ; \mathrm{df}=1 ; P=0.04$ ). Conclusion. Our findings confirmed the contribution of GCKR rs780093 polymorphism to TG metabolism and demonstrated GCKR rs780093 as a risk factor of CHD in individuals aged 65 and older.

\section{Introduction}

Coronary heart disease (CHD) is one of the leading causes of morbidity and death in the developed countries, and its prevalence is increasing rapidly in the developing countries [1]. CHD has become a major health burden in the world [2]. CHD is a type of complex disease caused by both environmental and genetic factors, as well as interactions among these factors [3]. CHD may lead to severe events, including sudden cardiac death (SCD) or acute myocardial infarction (AMI). CHD is heritable [4-6]. Although genetic factors are estimated to account for about $30-60 \%$ of the CHD risk $[7,8]$, the pathogenesis of CHD is still not fully understood.

Aberrant levels of blood lipids and glucose are risk factors of CHD $[9,10]$. Elevated triglyceride (TG) levels were shown to be associated with increased risk of $\mathrm{CHD}$ and other cardiovascular events [11]. The GCKR locus is the strongest completely novel locus implicated in TG metabolism [12].
Interestingly, GCKR is also associated with the risk of cardiovascular disease [13].

Located on chromosome 2p23, GCKR gene consists of 19 exons and encodes a protein comprising 625 amino acids [14]. GCKR is a susceptibility gene for type 2 diabetes mellitus [15]. GCKR gene product inhibits glucokinase in liver and pancreatic islet cells by binding noncovalently to form an inactive complex with glucokinase and thus plays a pivotal role in glucose homeostasis $[16,17]$. Recent genome-wide association studies (GWAS) have identified that GCKR is important for modulating serum TG [18] or fasting blood glucose levels. CHD risk is strongly and independently associated with aberrant glucose metabolism $[19,20]$. Furthermore, animal studies have confirmed that perturbation of the GCKR metabolic pathways may increase serum TG concentrations [21, 22].

GCKR rs780093 (A/G) polymorphism is an intronic SNP. A recent study discovered that GCKR rs780093 was associated with TG levels in Europeans [15]. Since most studies have 
been conducted in Europeans, one goal of our study is to confirm the correlation of GCKR rs780093 polymorphism and TG levels in Han Chinese population.

In the present study, we collected $568 \mathrm{CHD}$ individuals and 494 non-CHD controls from Ningbo in Eastern China and performed a case-control study to investigate the contribution of GCKR rs780093 polymorphism to the risk of CHD and TG levels in Han Chinese population.

\section{Methods and Materials}

2.1. Sample Collection. A total of 1062 unrelated patients were recruited from the Ningbo Lihuili Hospital between September 2011 and May 2013. Our study consisted of 568 CHD cases (mean age: $61.66 \pm 9.35$ ) and 494 non-CHD controls (mean age: $57.96 \pm 9.90$ ). All patients were examined by standardized coronary artery angiographic according to Seldinger's method [23]. The diagnostic results were judged by at least two independent cardiologists. According to the angiographic results, $\mathrm{CHD}$ cases were defined as the diameter degree of stenosis $\geq 50 \%$ in any of the main coronary arteries. In addition, patients with a history of prior angioplasty or coronary artery bypass surgery were considered as CHD cases. Non-CHD controls had $<50 \%$ stenosis of any coronary artery or no history of the atherosclerotic vascular disease. The individuals with congenital heart disease or cardiomyopathy, liver, or kidney diseases were not included in the case or control groups. The blood samples were collected from the cases and controls in a fasting state and treated by the same investigators. Then, blood samples were collected in $3.2 \%$ citrate sodium-treated tubes and stored at $-80^{\circ} \mathrm{C}$ until analyzed. Serum triglycerides and total cholesterol were determined using an automatic analyzer (Hitachi 7060, Hitachi, Tokyo, Japan) within one month of sample collection. The study protocol was approved by the Ethics Committee of Lihuili Hospital in Ningbo. All participants signed the informed consent that included genetic association studies.

2.2. SNP Genotyping. Genomic DNA was isolated from peripheral blood lymphocytes by the nucleic acid extraction automatic analyzer (Lab-Aid 820, Xiamen, China). DNA concentration was quantified using the PicoGreen double strand DNA Quantification Kit (Molecular Probes Inc., USA). PCR was performed on the GeneAmp PCR System, Veriti 96-well sample block module (Applied Biosystems, $\mathrm{AB}$, USA). PCRs were set up as follows: $0.25 \mu \mathrm{L}$ in each of the three primers (Table 1); $6 \mu \mathrm{L}$ SYBR Green I; $3.25 \mu \mathrm{L}$ $\mathrm{ddH}_{2} \mathrm{O}$; and $2 \mu \mathrm{L}$ DNA. PCR procedure was set to start with an initial denaturation at $95^{\circ} \mathrm{C}$ for $30 \mathrm{sec}$, followed by 40 cycles of a three-step amplification, including denaturation at $95^{\circ} \mathrm{C}$ for $30 \mathrm{sec}, 30 \mathrm{sec}$ at $59^{\circ} \mathrm{C}$ for annealing, $30 \mathrm{sec}$ at $72^{\circ} \mathrm{C}$ for primer extension, and a final extension for $30 \mathrm{sec}$ at $72^{\circ} \mathrm{C}$. Genotyping of the PCR products was performed using melting temperature shift (Tm shift) according to the manufacturer's instructions [24]. Tm-shift method uses two allele-specific primers and one reverse primer to amplify the polymorphic region encoding the targeted variant (Figure 1 and Table 1).

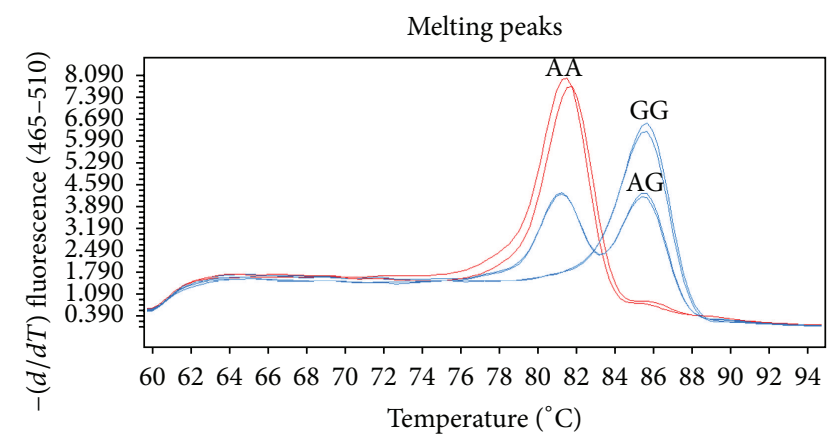

FIGURE 1: Genotyping of GCKR rs780093 using Tm-shift method. GG genotype has higher Tm value, namely, the $5^{\prime}$ end to join long sequences of alleles homozygous. AA genotype has lower Tm value, namely, the $5^{\prime}$ end to join short sequences of alleles homozygous. AG has the mixed curves with low and high Tm values.

2.3. Statistical Analyses. Hardy-Weinberg equilibrium (HWE) was analyzed by the Arlequin program (version 3.5) [25]. Genotype distribution and allele frequencies between $\mathrm{CHD}$ cases and non-CHD controls were determined using Clump22 software with 10,000 Monte Carlo simulations [26]. Power analysis was performed using the Power and Sample Size Calculation Software [27]. The odds ratio (OR) and 95\% confidence interval (CI) were assessed with an online program (http://faculty.vassar.edu/lowry/odds $2 \times 2 . h t m l)$. The logistic regression analysis was performed between genotype and degree of coronary stenosis using SAS statistical software. One-way ANOVA test was used to test the correlation between genotype and TG levels. Significant differences of all tests were indicated by values of $P<0.05$.

\section{Results}

Baseline characteristics of the study populations were shown in Table 2. Genotype distribution of rs780093 met HWE for both CHD cases and controls $(P>0.05$, Table 3$)$. Genotype and allele frequencies of GCKR rs780093 polymorphism were shown in Table 3. Figure 2 demonstrated the three genotypes (AA, AG, and GG) of GCKR rs780093 polymorphism by Tm-shift analysis. Our results showed that rs780093 was not associated with $\mathrm{CHD}$ in the whole samples (Table 3 ), the gender-stratified samples (Table 4). Association under the dominant and recessive models was also unable to present a significant association of rs780093 with CHD (Table 5). Recent study in American population has shown that senior individuals ( $\geq 65$ years of age) are eligible for secondary prevention of CHD [28]. Since age is an immutable risk factor of $\mathrm{CHD}$, we performed an age-stratified analysis to study whether age influences the contribution of rs780093 to the risk of CHD. As shown in Table 6, our subgroup analysis by age indicated a significant association between rs780093 and $\mathrm{CHD}$ among individuals aged 65 or older (genotype: $\chi^{2}=6.86 ; \mathrm{df}=2 ; P=0.03$; allele: $\mathrm{OR}=1.38 ; 95 \% \mathrm{CI}=1.01-1.89$; $\left.\chi^{2}=4.11 ; \mathrm{df}=1 ; P=0.04\right)$.

Our study did not observe a gender difference for the association of rs780093 with $\mathrm{CHD}(P>0.05)$. A further 
TABLE 1: Genotyping primers ${ }^{\mathrm{a}}$.

\begin{tabular}{lcr}
\hline SNP & Primer type & Primer sequence \\
\hline \multirow{2}{*}{ rs780093 } & GCKR-gF & $5^{\prime}$-gcgggcagggcggcCCCAAGCAAGAGCCCCCg-3 $^{\prime}$ \\
& GCKR-aF & $5^{\prime}$-gattaccgCCCAAGCAAGAGCCCCCa- ${ }^{\prime}$ \\
& GCKR-R & $5^{\prime}$-CCTGTCCTGTGGTTCTTGCAAATGC-3 $^{\prime}$ \\
\hline
\end{tabular}

${ }^{a}$ GCKR-gF and GCKR-aF stand for $g$ and a allele-specific primers, respectively. GCKR-R denotes reverse primer.

TABLE 2: Patients' main clinical characteristics ${ }^{\mathrm{a}}$.

\begin{tabular}{lcc}
\hline Characteristics & Cases $(n=568)$ & Controls $(n=494)$ \\
\hline Men $(\%)$ & $405(71.3)$ & $270(54.7)$ \\
Age $($ years, mean \pm SD) & $61.66 \pm 9.35$ & $57.96 \pm 9.90$ \\
TC $(\mathrm{mmol} / \mathrm{L})$ & $4.28 \pm 1.13$ & $4.33 \pm 0.98$ \\
TG $(\mathrm{mmol} / \mathrm{L})$ & $1.68 \pm 1.08$ & $1.57 \pm 0.96$ \\
\hline
\end{tabular}

${ }^{\mathrm{a}} \mathrm{TC}$ stands for total cholesterol; TG denotes triglycerides.

genetic test under the dominant and recessive genetic models did not yield a significant result. A further subgroup analysis by age showed a significant association of rs780093 with $\mathrm{CHD}$ in the senior individuals (over 65 years old) under the dominant model (Table 7, $P=0.01$, OR $=2.03$, and $95 \%$ $\mathrm{CI}=1.26-3.27$ ).

According to the angiographic results, we defined the severity of CHD into three stages, including one, two, and three or more coronary arteries with $\geq 50 \%$ stenosis. Logistic regression analysis showed no significant association between the stages of coronary artery stenosis and rs780093 (Table 8).

In light of previous association between TG concentrations and rs780093 in European populations, we performed a similar correlation test in Han Chinese population. Our data confirmed that rs780093-AA genotype predicted a much higher TG level than other genotypes in Han Chinese population $(P=0.0016$, Figure 2$)$.

\section{Discussion}

Our case-control study is aimed to explore the contribution of rs780093 to both the risk of CHD and TG level in Han Chinese population. Previous studies have demonstrated that the levels of TG and low-density lipoprotein cholesterol were independent heritable risk factors for cardiovascular diseases [29]. Some of the risk genes for CHD may influence their function by regulating or interacting with these factors. TG is composed of glycerol and fatty acids; circulating levels of fatty acids had been linked to the risk of CHD [30, 31]. Previous meta-analysis has shown a strong association of rs780093 with the level of fatty acids in Europeans [32]. And rs780093 A/A genotype was associated with both elevated circulating levels of fatty acids [32] and higher TG levels in multiple populations including Europeans [15, 33], Americans [33], and African Americans [34]. Consistent with previous findings, our results revealed that rs780093 was significantly associated with TG in Han Chinese population. In GCKR, rs780093 is in strong linkage disequilibrium (LD) with rs1260326 (Pro446Leu) in both YRI $\left(r^{2}=0.65\right)$ and CEU HapMap populations $\left(r^{2}=0.93\right)$. A previous study

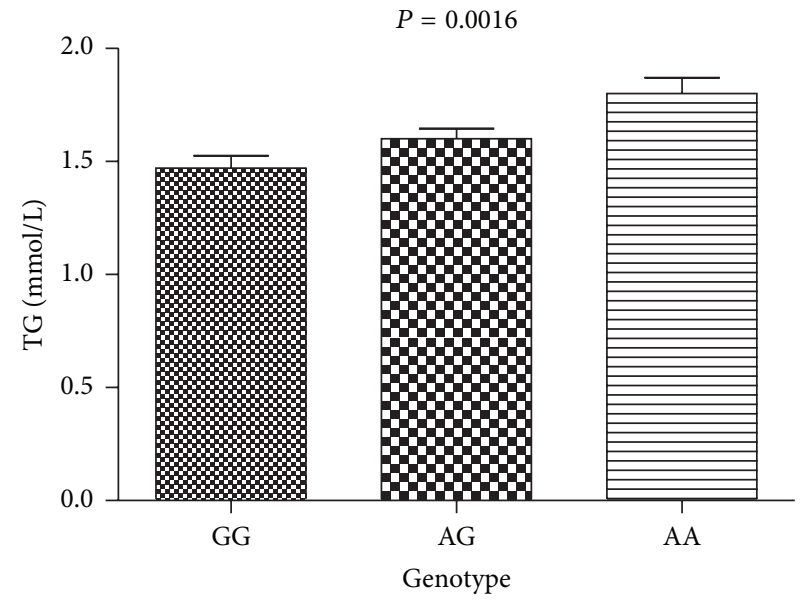

FIGURE 2: Association between TG concentrations and GCKR rs780093 polymorphism. TG concentrations are expressed as mean \pm SEM.

in European-ancestry individuals showed that rs1260326 had been consistently associated with elevated triglycerides and reduced fasting plasma glucose and reduced fasting plasma glucose $[35,36]$. P446L of GCKR gene had shown to reduce inhibitory activity toward glucokinase, which would be predicted to increase glycolytic level and production of malonyl-CoA, a key substrate for TG synthesis and de novo lipogenesis [36]. Future study is warranted to investigate whether GCKR variants are able to increase the availability of substrate for de novo lipogenesis.

Early-onset CHD is defined if there is acute coronary syndrome (myocardial infarction or unstable angina), a revascularization process, or a positive functional imaging study at or before the age of 50 years in men or 55 years in women [37]. Most CHD occurs in individuals aged over 65; only $5-10 \%$ of CHD occurs in younger patients [2]. Although we cannot observe a significant association between rs780093 of GCKR gene and early-onset CHD, there is a significant association of rs780093 with CHD patients after 65.

According to the angiographic results, CHD patients were simply divided into three subgroups by the number of coronary arteries with stenosis. This classification method of CHD might not accurately reflect the severity of CHD, since the percentage of stenosis is not taken into account for the severity scale. This limitation in the classification may lead to a lack of correlation between rs780093 and the severity of CHD. A better parameter is needed to define the extent of coronary artery stenosis. 
TABLE 3: Genotype distribution and allele frequencies of rs780093 in cases and controls ${ }^{\mathrm{a}}$.

\begin{tabular}{lccccccccrrr}
\hline \multirow{2}{*}{ rs780093 } & \multicolumn{3}{c}{ Genotype (counts) } & $\chi^{2}$ & $P(\mathrm{df}=2)$ & HWE & \multicolumn{2}{c}{ Allele (counts) } & $\chi^{2}$ & $P(\mathrm{df}=1)$ & OR $(95 \% \mathrm{CI})$ \\
& $\mathrm{GG}$ & AG & AA & & & & $\mathrm{G}$ & $\mathrm{A}$ & & \\
\hline Cases $(n=568)$ & 125 & 297 & 146 & & & 0.28 & 547 & 589 & & \\
Controls $(n=494)$ & 109 & 252 & 133 & 0.23 & 0.89 & 0.65 & 470 & 518 & 0.07 & 0.79 & $1.02(0.86-1.21)$ \\
\hline
\end{tabular}

${ }^{\mathrm{a}}$ HWE: Hardy-Weinberg equilibrium; OR: odds ratio; CI: confidence interval.

TABLE 4: A breakdown association test between cases and controls by gender ${ }^{\mathrm{a}}$.

\begin{tabular}{|c|c|c|c|c|c|c|c|c|c|c|c|c|}
\hline \multirow{2}{*}{ Gender } & \multirow{2}{*}{ rs780093 } & \multicolumn{3}{|c|}{ Genotype (counts) } & \multirow[t]{2}{*}{$\chi^{2}$} & \multirow[t]{2}{*}{$P(\mathrm{df}=2)$} & \multirow[t]{2}{*}{ HWE } & \multicolumn{2}{|c|}{ Allele (counts) } & \multirow[t]{2}{*}{$\chi^{2}$} & \multirow[t]{2}{*}{$P(\mathrm{df}=1)$} & \multirow[t]{2}{*}{ OR (95\% CI) } \\
\hline & & GG & $\mathrm{AG}$ & AA & & & & $\mathrm{G}$ & $\mathrm{A}$ & & & \\
\hline \multirow{2}{*}{ Male } & Case $(N=405)$ & 93 & 211 & 101 & & & 0.43 & 397 & 413 & & & \\
\hline & Controls $(N=270)$ & 63 & 138 & 69 & 0.06 & 0.97 & 0.81 & 264 & 276 & 0.00 & 0.96 & $1.01(0.81-1.25)$ \\
\hline \multirow{2}{*}{ Female } & Case $(N=163)$ & 32 & 86 & 45 & & & 0.53 & 150 & 176 & & & \\
\hline & Controls $(N=224)$ & 46 & 114 & 64 & 0.13 & 0.94 & 0.79 & 206 & 242 & 0.00 & 0.99 & $1.00(0.75-1.33)$ \\
\hline
\end{tabular}

${ }^{a}$ HWE: Hardy-Weinberg equilibrium; OR: odds ratio; CI: confidence interval.

TABLE 5: Association of rs780093 with CHD under the dominant and recessive models ${ }^{\mathrm{a}}$.

\begin{tabular}{lcccccccrrr}
\hline \multirow{2}{*}{ rs780093 } & \multicolumn{2}{c}{ Dominant } & $\chi^{2}$ & $P(\mathrm{df}=1)$ & OR $(95 \% \mathrm{CI})$ & \multicolumn{2}{c}{ Recessive } & $\chi^{2}$ & $P(\mathrm{df}=1)$ & OR $(95 \% \mathrm{CI})$ \\
& $\mathrm{GG}+\mathrm{GA}$ & $\mathrm{AA}$ & & & & $\mathrm{GG}$ & $\mathrm{GA}+\mathrm{AA}$ & & \\
\hline Cases & 422 & 146 & & & & 125 & 443 & & \\
Controls & 361 & 133 & 0.20 & 0.65 & $1.06(0.81-1.40)$ & 109 & 385 & 0.00 & 0.98 & $1.00(0.75-1.33)$ \\
\hline
\end{tabular}

${ }^{\mathrm{a}} \mathrm{OR}$ : odds ratio; CI: confidence interval.

TABLE 6: A breakdown association test between cases and controls by age $\mathrm{e}^{\mathrm{a}}$.

\begin{tabular}{|c|c|c|c|c|c|c|c|c|c|c|c|c|}
\hline \multirow{2}{*}{ Age } & \multirow{2}{*}{ rs780093 } & \multicolumn{3}{|c|}{ Genotype (counts) } & \multirow[t]{2}{*}{$\chi^{2}$} & \multirow[t]{2}{*}{$P(\mathrm{df}=2)$} & \multirow[t]{2}{*}{ HWE } & \multicolumn{2}{|c|}{ Allele (counts) } & \multirow[t]{2}{*}{$\chi^{2}$} & \multirow[t]{2}{*}{$P(\mathrm{df}=1)$} & \multirow[t]{2}{*}{ OR (95\% CI) } \\
\hline & & GG & GA & AA & & & & G & $\mathrm{A}$ & & & \\
\hline \multirow{2}{*}{$\leq 55$} & Cases $(n=138)$ & 25 & 73 & 40 & & & 0.49 & 123 & 153 & & & \\
\hline & Controls $(n=186)$ & 44 & 100 & 42 & 2.44 & 0.30 & 0.38 & 188 & 184 & 2.26 & 0.13 & $0.79(0.58-1.08)$ \\
\hline \multirow{2}{*}{$55-65$} & Cases $(n=200)$ & 52 & 94 & 54 & & & 0.40 & 198 & 202 & & & \\
\hline & Controls $(n=187)$ & 43 & 96 & 48 & 0.79 & 0.67 & 0.77 & 182 & 192 & 0.05 & 0.82 & $1.03(0.78-1.37)$ \\
\hline \multirow{2}{*}{$\geq 65$} & Cases $(n=231)$ & 49 & 130 & 52 & & & 0.07 & 228 & 234 & & & \\
\hline & Controls $(n=121)$ & 22 & 56 & 43 & 6.86 & 0.03 & 0.71 & 100 & 142 & 4.11 & 0.04 & $1.38(1.01-1.89)$ \\
\hline
\end{tabular}

${ }^{a}$ HWE: Hardy-Weinberg equilibrium; OR: odds ratio; CI: confidence interval.

TABLE 7: A breakdown association of rs780093 with CHD by age under the dominant and recessive models ${ }^{\mathrm{a}}$.

\begin{tabular}{|c|c|c|c|c|c|c|c|c|c|c|c|}
\hline \multirow{2}{*}{ Age } & \multirow{2}{*}{ rs780093 } & \multicolumn{2}{|c|}{ Dominant } & \multirow[t]{2}{*}{$\chi^{2}$} & \multirow[t]{2}{*}{$P(\mathrm{df}=1)$} & \multirow[t]{2}{*}{ OR (95\% CI) } & \multicolumn{2}{|c|}{ Recessive } & \multirow[t]{2}{*}{$\chi^{2}$} & \multirow[t]{2}{*}{$P(\mathrm{df}=1)$} & \multirow[t]{2}{*}{ OR (95\% CI) } \\
\hline & & $\mathrm{GG}+\mathrm{GA}$ & AA & & & & GG & $\mathrm{GA}+\mathrm{AA}$ & & & \\
\hline \multirow{2}{*}{$\leq 55$} & Case $(N=138)$ & 98 & 40 & & & & 25 & 113 & & & \\
\hline & Controls $(N=186)$ & 144 & 42 & 1.72 & 0.19 & $0.71(0.43-1.18)$ & 44 & 142 & 1.45 & 0.23 & $0.71(0.41-1.24)$ \\
\hline \multirow{2}{*}{$55-65$} & Case $(N=200)$ & 146 & 54 & & & & 52 & 148 & & & \\
\hline & Controls $(N=187)$ & 139 & 48 & 0.08 & 0.77 & $0.93(0.59-1.47)$ & 43 & 144 & 0.47 & 0.49 & $1.18(0.74-1.87)$ \\
\hline \multirow{2}{*}{$\geq 65$} & Case $(N=231)$ & 179 & 52 & & & & 49 & 182 & & & \\
\hline & Controls $(N=121)$ & 78 & 43 & 6.84 & 0.01 & $2.03(1.26-3.27)$ & 22 & 99 & 0.45 & 0.50 & $1.21(0.69-2.12)$ \\
\hline
\end{tabular}

${ }^{\mathrm{a}}$ OR: odds ratio; CI: confidence interval.

TABLE 8: Logistic regression analysis between rs780093 and the severity of CHD.

\begin{tabular}{lccccc}
\hline & Non-CHD controls & One affected artery & Two affected arteries & $\geq$ Three affected arteries & Association with rs780093 $(P$ value $)$ \\
\hline Male & 270 & 154 & 111 & 137 & 0.98 \\
Female & 224 & 83 & 36 & 40 & 0.47 \\
Total & $\mathbf{4 9 4}$ & $\mathbf{2 3 7}$ & $\mathbf{1 4 7}$ & $\mathbf{1 7 7}$ & $\mathbf{0 . 7 4}$ \\
\hline
\end{tabular}


In the present study, the allele frequency of $\mathrm{G}(47.8 \%)$ is similar with that in HapMap CHB population (42.9\%). We also observed a large ethnic difference of rs780093 in European (60.6\%), Sub-Saharan African (89.8\%), and Chinese population (47.8\%) according to rs780093 frequency report in the HapMap project database. Although a total of 1062 individuals were recruited in the present study, our association of rs780093 has a 55\% power, suggesting that our sample size may be not enough. Meanwhile, all the $P$ values in the case-control study are not corrected by the number of tests; thus, we cannot exclude the possibility of false positive results in our findings.

In summary, GCKR rs780093 was significantly associated with the risk of CHD in Han Chinese population aged 65 or older and significantly lower TG levels. In addition, we observed the significant association between rs780093 and $\mathrm{CHD}$ after the age of 65 under the dominant model.

\section{Authors' Contribution}

Jiangfang Lian and Jian Guo contributed equally to this work.

\section{Acknowledgments}

The research was supported by the Grants from the National Natural Science Foundation of China (31100919 and 81371469), Natural Science Foundation of Zhejiang Province (LR13H020003 and LY13H020008), K.C. Wong Magna Fund in Ningbo University, Advanced Key Scientific and Technological Programs of Ningbo (2011C51001), Fund of Ningbo Science and Technology Innovation Team (2011B82015), Natural Science Foundation of Ningbo City (2011A610037), Ningbo Social Development Research Projects (2012C50032), Ningbo Personnel Training Project (first level), and the Project of Ningbo Medicine and Science (2009A02).

\section{References}

[1] "WHO publishes definitive atlas on global heart disease and stroke epidemic," Indian Journal of Medical Sciences, vol. 58, pp. 405-406, 2004.

[2] V. L. Roger, A. S. Go, D. M. Lloyd-Jones et al., "Executive summary: heart disease and stroke statistics-2012 update: a report from the American heart association," Circulation, vol. 125, no. 1, pp. 188-197, 2012.

[3] S. Maouche and H. Schunkert, "Strategies beyond genomewide association studies for atherosclerosis," Arteriosclerosis, Thrombosis, and Vascular Biology, vol. 32, no. 2, pp. 170-181, 2012.

[4] I. J. Kullo and K. Ding, "Mechanisms of disease: the genetic basis of coronary heart disease," Nature Clinical Practice Cardiovascular Medicine, vol. 4, no. 10, pp. 558-569, 2007.

[5] B. Mayer, J. Erdmann, and H. Schunkert, "Genetics and heritability of coronary artery disease and myocardial infarction," Clinical Research in Cardiology, vol. 96, no. 1, pp. 1-7, 2007.

[6] G. Pilia, W. Chen, A. Scuteri et al., "Heritability of cardiovascular and personality traits in 6,148 Sardinians," PLoS Genetics, vol. 2, no. 8, article el32, 2006.
[7] S. Zdravkovic, A. Wienke, N. L. Pedersen, M. E. Marenberg, A. I. Yashin, and U. de Faire, "Heritability of death from coronary heart disease: a 36-year follow-up of 20966 Swedish twins," Journal of Internal Medicine, vol. 252, no. 3, pp. 247-254, 2002.

[8] M. E. Marenberg, N. Risch, L. F. Berkman, B. Floderus, and U. De Faire, "Genetic susceptibility to death from coronary heart disease in a study of twins," The New England Journal of Medicine, vol. 330, no. 15, pp. 1041-1046, 1994.

[9] J. I. Cleeman, "Executive summary of the third report of the National Cholesterol Education Program (NCEP) expert panel on detection, evaluation, and treatment of high blood cholesterol in adults (adult treatment panel III)," Journal of the American Medical Association, vol. 285, no. 19, pp. 2486-2497, 2001.

[10] K. G. Alberti and P. Z. Zimmet, "Definition, diagnosis and classification of diabetes mellitus and its complications. Part 1: diagnosis and classification of diabetes mellitus provisional report of a WHO consultation," Diabetic Medicine, vol. 15, pp. 539-553, 1998.

[11] A. Patel, "Serum triglycerides as a risk factor for cardiovascular diseases in the Asia-Pacific region," Circulation, vol. 110, no. 17, pp. 2678-2686, 2004.

[12] R. Saxena, B. F. Voight, V. Lyssenko et al., "Genome-wide association analysis identifies loci for type 2 diabetes and triglyceride levels," Science, vol. 316, no. 5829, pp. 1331-1336, 2007.

[13] C. T. Johansen, S. Kathiresan, and R. A. Hegele, "Genetic determinants of plasma triglycerides," Journal of Lipid Research, vol. 52, no. 2, pp. 189-206, 2011.

[14] E. van Schaftingen, "A protein from rat liver confers to glucokinase the property of being antagonistically regulated by fructose 6-phosphate and fructose 1-phosphate," European Journal of Biochemistry, vol. 179, no. 1, pp. 179-184, 1989.

[15] A. T. Kraja, D. Vaidya, J. S. Pankow et al., "A bivariate genomewide approach to metabolic syndrome: STAMPEED consortium," Diabetes, vol. 60, no. 4, pp. 1329-1339, 2011.

[16] J. Grimsby, J. W. Coffey, M. T. Dvorozniak et al., "Characterization of glucokinase regulatory protein-deficient mice," The Journal of Biological Chemistry, vol. 275, no. 11, pp. 7826-7831, 2000.

[17] D. Farrelly, K. S. Brown, A. Tieman et al., "Mice mutant for glucokinase regulatory protein exhibit decreased liver glucokinase: a sequestration mechanism in metabolic regulation," Proceedings of the National Academy of Sciences of the United States of America, vol. 96, no. 25, pp. 14511-14516, 1999.

[18] J. Dupuis, C. Langenberg, I. Prokopenko et al., "New genetic loci implicated in fasting glucose homeostasis and their impact on type 2 diabetes risk," Nature Genetics, vol. 42, pp. 105-116, 2010.

[19] K. Shaye, T. Amir, S. Shlomo, and S. Yechezkel, "Fasting glucose levels within the high normal range predict cardiovascular outcome," American Heart Journal, vol. 164, pp. 111-116, 2012.

[20] M. Li, B. McCulloch, and R. McDermott, "Metabolic syndrome and incident coronary heart disease in Australian indigenous populations," Obesity, vol. 20, pp. 1308-1312, 2012.

[21] R. M. O’Doherty, D. L. Lehman, S. Télémaque-Potts, and C. B. Newgard, "Metabolic impact of glucokinase overexpression in liver: lowering of blood glucose in fed rats is accompanied by hyperlipidemia," Diabetes, vol. 48, no. 10, pp. 2022-2027, 1999.

[22] T. Ferre, E. Riu, F. Bosch, and A. Valera, "Evidence from transgenic mice that glucokinase is rate limiting for glucose utilization in the liver," The FASEB Journal, vol. 10, no. 10, pp. 1213-1218, 1996. 
[23] Z. C. J. Higgs, D. A. L. Macafee, B. D. Braithwaite, and C. A. Maxwell-Armstrong, "The Seldinger technique: 50 years on," The Lancet, vol. 366, no. 9494, pp. 1407-1409, 2005.

[24] S. Germer and R. Higuchi, "Single-tube genotyping without oligonucleotide probes," Genome Research, vol. 9, no. 1, pp. 7278, 1999.

[25] L. Excoffier and H. E. L. Lischer, "Arlequin suite ver 3.5: a new series of programs to perform population genetics analyses under Linux and Windows," Molecular Ecology Resources, vol. 10, no. 3, pp. 564-567, 2010.

[26] P. C. Sham and D. Curtis, "Monte Carlo tests for associations between disease and alleles at highly polymorphic loci," Annals of Human Genetics, vol. 59, no. 1, pp. 97-105, 1995.

[27] W. D. Dupont and W. D. Plummer Jr., "Power and sample size calculations. A review and computer program," Controlled Clinical Trials, vol. 11, no. 2, pp. 116-128, 1990.

[28] M. A. Williams, J. L. Fleg, P. A. Ades et al., "Secondary prevention of coronary heart disease in the elderly (with emphasis on patients $\geq 75$ years of age): an american heart association scientific statement from the council on clinical cardiology subcommittee on exercise, cardiac rehabilitation, and prevention," Circulation, vol. 105, no. 14, pp. 1735-1743, 2002.

[29] S. Kathiresan, A. K. Manning, S. Demissie et al., "A genomewide association study for blood lipid phenotypes in the Framingham Heart Study," BMC Medical Genetics, vol. 8, supplement 1, article S17, 2007.

[30] E. Warensjö, J. Sundström, B. Vessby, T. Cederholm, and U. Risérus, "Markers of dietary fat quality and fatty acid desaturation as predictors of total and cardiovascular mortality: a population-based prospective study," American Journal of Clinical Nutrition, vol. 88, no. 1, pp. 203-209, 2008.

[31] J. A. Simon, M. L. Hodgkins, W. S. Browner, J. M. Neuhaus, J. T. Bernert Jr., and S. B. Hulley, "Serum fatty acids and the risk of coronary heart disease," American Journal of Epidemiology, vol. 142, no. 5, pp. 469-476, 1995.

[32] J. H. Wu, R. N. Lemaitre, A. Manichaikul et al., "Genomewide association study identifies novel loci associated with concentrations of four plasma phospholipid fatty acids in the de novo lipogenesis pathway: results from the Cohorts for Heart and Aging Research in Genomic Epidemiology (CHARGE) consortium," Circulation, vol. 6, pp. 171-183, 2013.

[33] S. Kathiresan, C. J. Willer, G. M. Peloso et al., "Common variants at 30 loci contribute to polygenic dyslipidemia," Nature Genetics, vol. 41, no. 1, pp. 56-65, 2009.

[34] M. E. Keebler, R. C. Deo, A. Surti et al., "Fine-mapping in African Americans of 8 recently discovered genetic loci for plasma lipids: the Jackson Heart Study," Circulation, vol. 3, no. 4, pp. 358-364, 2010.

[35] M. Orho-Melander, O. Melander, C. Guiducci et al., "Common missense variant in the glucokinase regulatory protein gene is associated with increased plasma triglyceride and C-reactive protein but lower fasting glucose concentrations," Diabetes, vol. 57, no. 11, pp. 3112-3121, 2008.

[36] N. L. Beer, N. D. Tribble, L. J. McCulloch et al., "The P446L variant in GCKR associated with fasting plasma glucose and triglyceride levels exerts its effect through increased glucokinase activity in liver," Human Molecular Genetics, vol. 18, no. 21, pp. 4081-4088, 2009.

[37] C. Ward-Caviness, C. Haynes, C. Blach, E. Dowdy, S. G. Gregory, S. H. Shah et al., "Gene-smoking interactions in multiple Rho-GTPase pathway genes in an early-onset coronary artery disease cohort," Human Genetics, vol. 132, no. 12, pp. 13711382, 2013. 


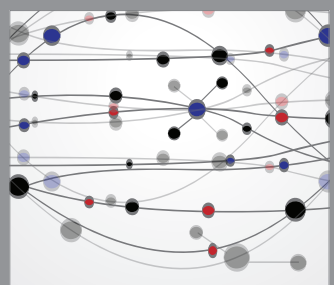

The Scientific World Journal
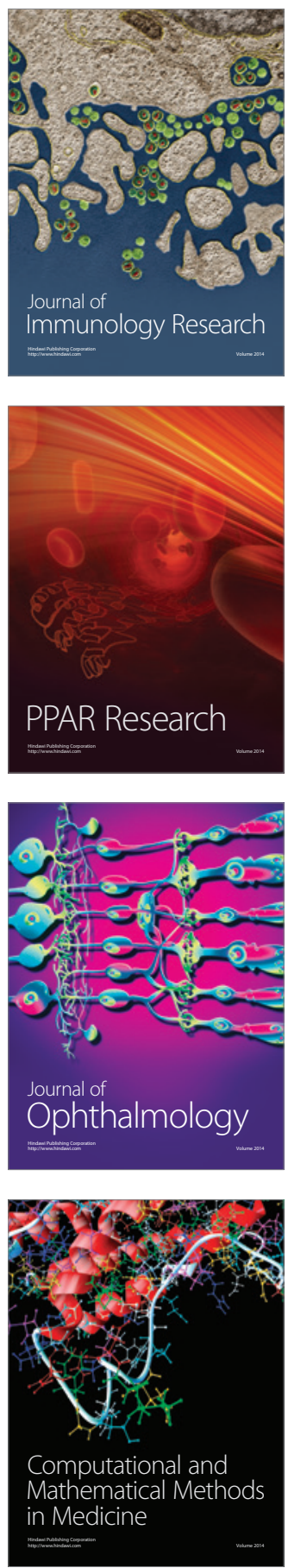

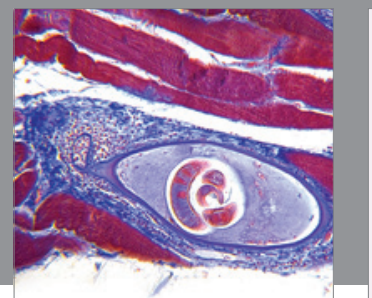

Gastroenterology

Research and Practice
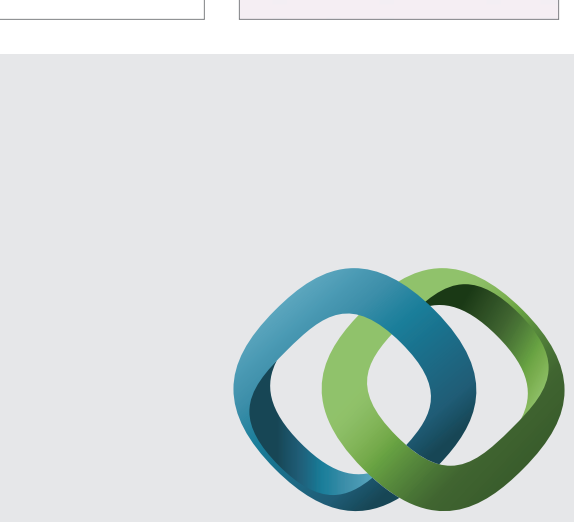

\section{Hindawi}

Submit your manuscripts at

http://www.hindawi.com
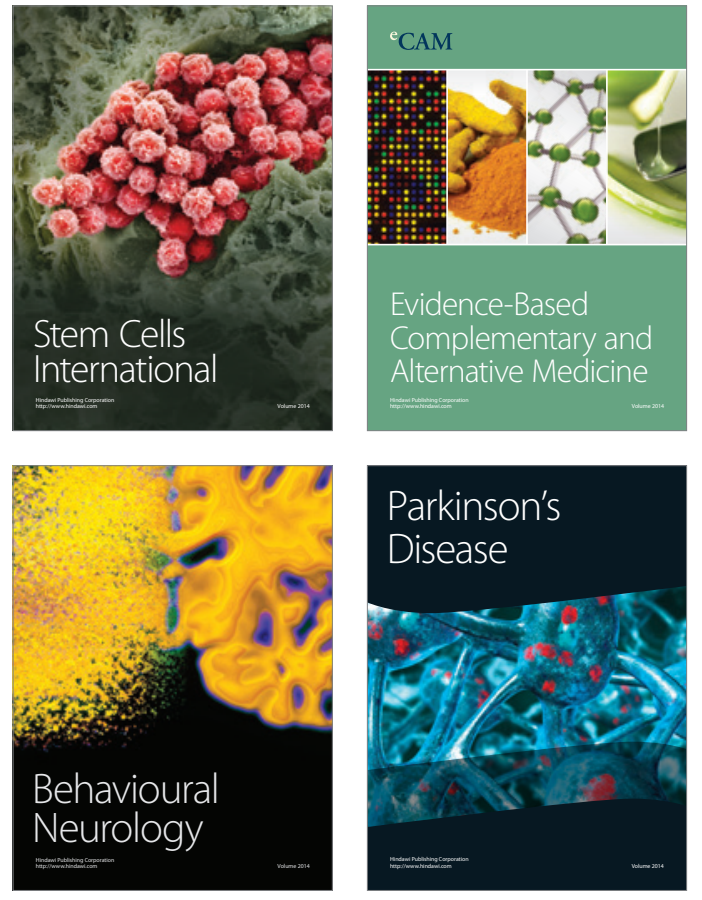
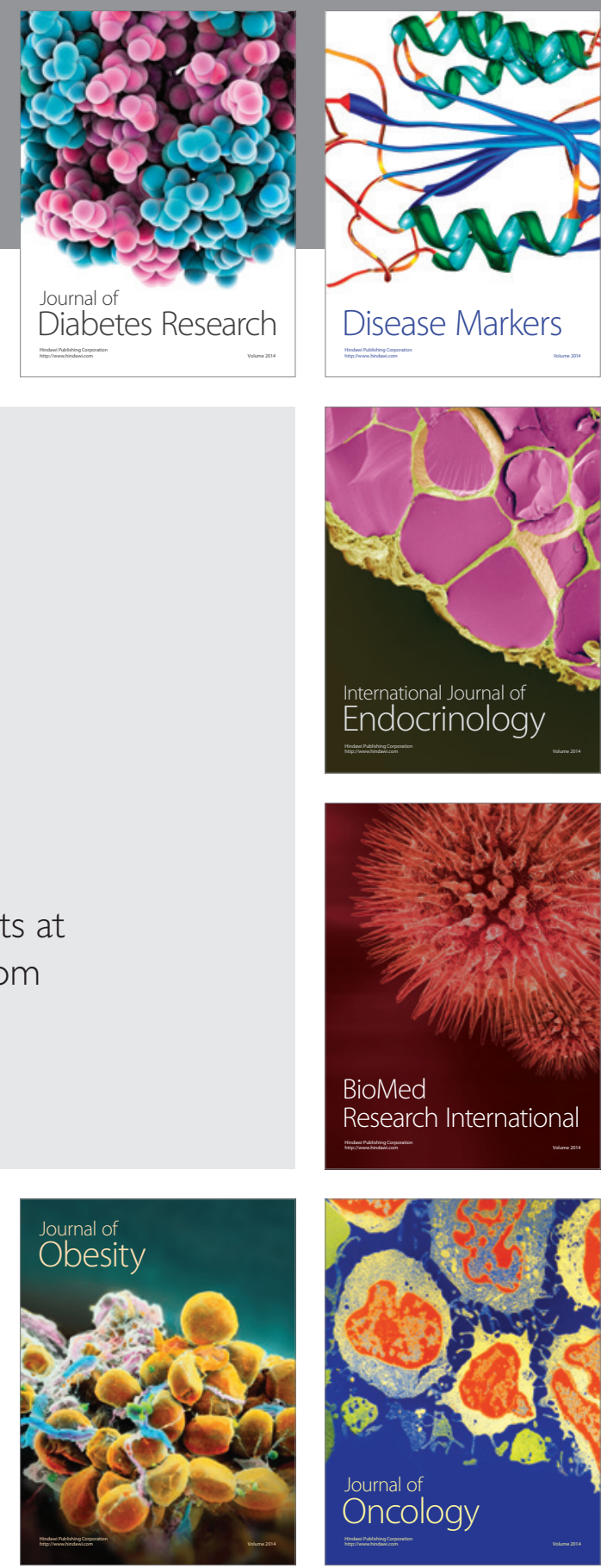

Disease Markers
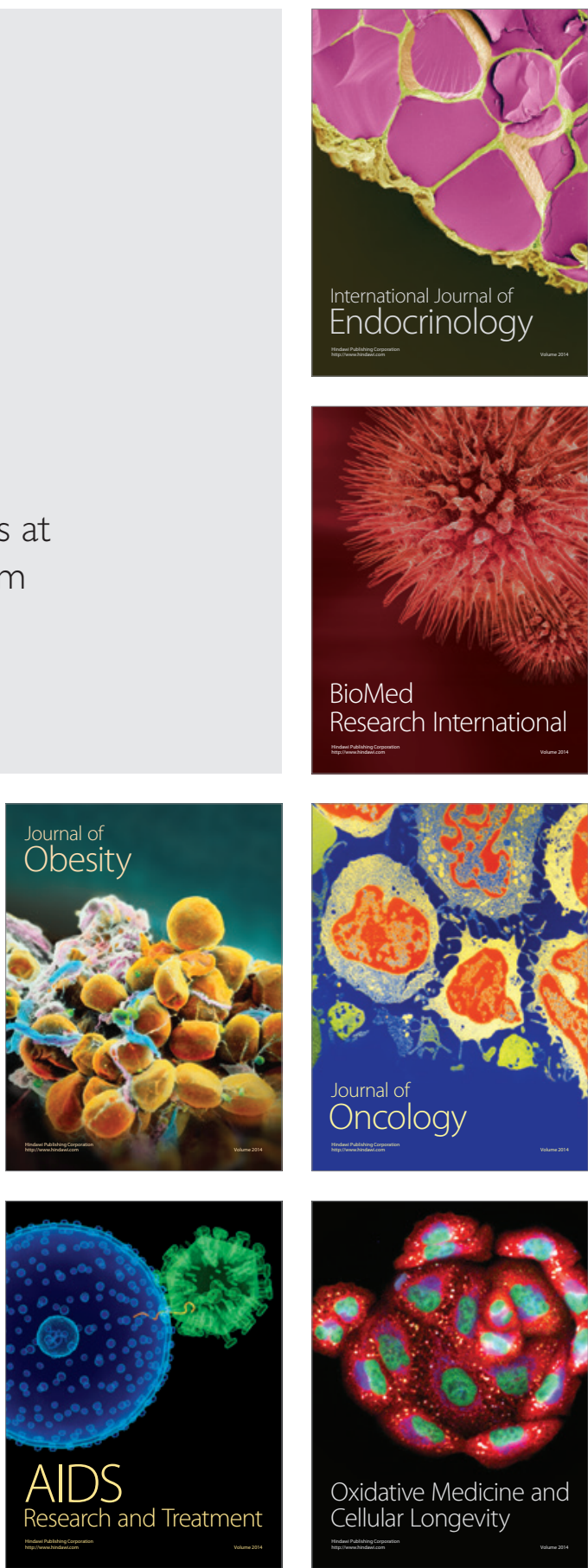